Gut and Liver, Vol. 12, No. 5, September 2018, pp. 481-482

\title{
Factors Predictive of Malignancy Potency in Pure Branch Duct Intraductal Papillary Mucinous Neoplasm of the Pancreas without Mural Nodules
}

\author{
Tae Hoon Lee \\ Department of Internal Medicine, Soonchunhyang University Cheonan Hospital, Soonchunhyang University School of Medicine, Cheonan, \\ Korea
}

See, "Predictors of Malignancy in "Pure" Branch-Duct Intraductal Papillary Mucinous Neoplasm of the Pancreas without Enhancing Mural Nodules on CT Imaging: A Nationwide Multicenter Study” by Tae-Hyeon Kim, et al. on page 583, Vol. 12. No. 5, 2018

Intraductal papillary mucinous neoplasm (IPMN) is a type of cystic pancreatic tumor with malignant potential. However, determining the risk of malignancy of IPMN, particularly branch duct type IPMN (BD-IPMN), is problematic. For this reason, the optimum management and follow up of BD-IPMN are debated although BD-IPMN is considered to have a low risk of malignancy. Revised guidelines or consensus statements on the management and follow-up of pancreatic cystic lesions have been issued by the American Gastroenterological Association, European associations, and the International Association of Pancreatology (IAP). ${ }^{1-5}$ The revised international consensus guidelines (ICG) ${ }^{2}$ of the IAP provide information on the characteristics, management, and recommended follow-up interval of BD-IPMN. Also, the 2018 European guideline provides greater detail on the indications for surgery and follow-up. ${ }^{4}$ However, the management and follow-up of pancreatic cystic tumors are still controversial. This study ${ }^{6}$ was based on the 2012 ICG for BD-IPMN, in which dilated main pancreatic ducts (MPDs) (5 to $9 \mathrm{~mm}$ in diameter) and cyst diameter $\geq 30 \mathrm{~mm}$ were reclassified as worrisome features. In the 2017 revised ICG, the highrisk stigmata of malignancy were revised as follows: obstructive jaundice in a patient with a cystic lesion of the pancreatic head, enhancing mural nodules (MNs) $>5 \mathrm{~mm}$, and MPD diameter $>10 \mathrm{~mm}$. Regarding worrisome features, dilated MNs $<5 \mathrm{~mm}$, lymphadenopathy, an increased serum level of CA19-9, and a cyst growth rate $>5 \mathrm{~mm} / 2$ years were newly included in the revised ICG. Despite of these guidelines, patients with IPMN require careful management and follow-up because of the dif- ficulty in predicting malignancy. Patients' performance status or age as well as malignant potency should be also considered before surgery.

This result will enable prediction of malignancy and assessment of risk factors in patients with pure BD-IPMN without MNs or solid masses on computed tomography (CT) imaging. ${ }^{6}$ Definite detection of true MNs or solid masses is usually difficult, particularly smaller lesions, therefore cyst size and the presence of ductal dilatation are still important findings for decision making on the appropriateness of surgery. A cyst diameter $>3$ $\mathrm{cm}$, the presence of enhancing MNs on CT, and MN $>5 \mathrm{~mm}$ on endoscopic ultrasonography (EUS) are independent predictors of malignancy in patients with BD-IPMNs in their previous report; ${ }^{7}$ these findings are similar to other reports. However, in this multicenter study, they tried to elucidate malignant potential and risk factors for malignancy in patients with pure BD-IPMN without enhancing MNs or solid masses on CT imaging. Clinically it may be difficult to decide to manage in these BD-IPMNs. In multivariate logistic regression analyses, the adjusted odds ratio (OR) for a cyst diameter $\geq 30 \mathrm{~mm}$ on abdominal CT imaging was 8.6 ( $p=0.001)$, while that for an MPD diameter $\geq 5 \mathrm{~mm}$ was 4.1 ( $p=0.01)$. Cyst size and MPD dilatation were associated with malignancy in pure BD-IPMN without MNs. ${ }^{6}$ Therefore, a cyst diameter $\geq 30 \mathrm{~mm}$ and MPD diameter $\geq 5 \mathrm{~mm}$ were associated with malignancy in pure BD-IPMNs, that is, those lacking enhanced MNs on CT. In pure type BD-IPMN, these findings may give a good direction for the plan of management.

Several guidelines suggest that patients with a cyst diameter

Correspondence to: Tae Hoon Lee

Division of Gastroenterology, Department of Internal Medicine, Soonchunhyang University Cheonan Hospital, Soonchunhyang University School of Medicine, 31 Suncheonhyang 6-gil, Dongnam-gu, Cheonan 31151, Korea

Tel: +82-41-570-3662, Fax: +82-41-574-5762, E-mail: thlee9@schmc.ac.kr pISSN 1976-2283 eISSN 2005-1212 https://doi.org/10.5009/gnl18305

@ This is an Open Access article distributed under the terms of the Creative Commons Attribution Non-Commercial License (http://creativecommons.org/licenses/by-nc/4.0) which permits unrestricted non-commercial use, distribution, and reproduction in any medium, provided the original work is properly cited. 
$>30 \mathrm{~mm}$ but without MNs on EUS should undergo close surveillance rather than surgery. However, in younger patients, surgical management should be recommended when the cyst is $>30$ $\mathrm{mm}$, even in the absence of other worrisome features. The 2018 European guidelines suggest surgery for cysts $>40 \mathrm{~mm}$ with MPD dilatation of 5 to $9.9 \mathrm{~mm}$, which are relative indications for surgery. ${ }^{4}$ Therefore, a cyst $>30 \mathrm{~mm}$ in diameter, irrespective of the patient's age, may be treated with surgery rather than surveillance if the performance status is suitable.

Regarding efficacy of EUS in the study of Kim et al., ${ }^{6}$ EUS was able to detect MNs that had been missed by CT in six patients (7.3\%), of whom two (33.3\%) had malignant IPMNs. The rate of malignant IPMNs was 5.3\% among the patients who had cysts without MNs on EUS. Multivariate logistic regression analyses revealed that the presence of MNs on EUS was a significant predictor of BD-IPMN malignancy (OR, 9.0; $\mathrm{p}=0.02$ ). ${ }^{6}$ Kobayashi et al. ${ }^{8}$ reported that BD-IPMN patients with MNs $<10$ $\mathrm{mm}$ in height should undergo observation rather than resection. However, according to the 2017 ICG, enhancing MNs >5 $\mathrm{mm}$ are high-risk stigmata, and enhancing MNs $<5 \mathrm{~mm}$ are a worrisome feature. Advanced EUS imaging modalities such as contrast-enhanced (CE)-EUS may enable detection of small cystic nodules or mass. The diagnostic capability of CE-EUS is increasing now. ${ }^{9,10}$ However, this study did not include patients with definite MNs; accordingly, cyst size and MPD dilatation results will facilitate treatment decision-making for BD-IPMN patients without definite MNs. Prior to decision making, EUS or CE-EUS also should be considered as a routine evaluation tool at risky patients like guidelines.

Nevertheless, why is the management of BD-IPMN controversial? As authors mentioned in their limitations, clinically many patients with BD-IPMN without surgery were not included due to the retrospective enrollment of surgically confirmed patients. Second, EUS or EUS-guided fine needle aspiration analysis was limited in this study. ${ }^{6}$ These two limitations may have led to selection bias. However, these limitations are likely shared by other retrospective studies because BD-IPMN malignancy can only be definitively diagnosed by pathologic testing.

In conclusion, the optimum management of BD-IPMN is still difficult, as most prior studies were retrospective analyses of patients who underwent surgery. Further large-scale prospective studies using various imaging modalities for predicting malignancy are warranted. Furthermore, the detection rate of malignancy will increase as imaging technologies advance; at present, multiple imaging modalities should be considered to increase the detection rate of malignancy.

\section{CONFLICTS OF INTEREST}

No potential conflict of interest relevant to this article was reported.

\section{ACKNOWLEDGEMENTS}

This work was supported by the Soonchunhyang University Research Fund.

\section{REFERENCES}

1. Tanaka M, Fernández-del Castillo C, Adsay V, et al. International consensus guidelines 2012 for the management of IPMN and MCN of the pancreas. Pancreatology 2012;12:183-197.

2. Tanaka M, Fernández-Del Castillo C, Kamisawa T, et al. Revisions of international consensus Fukuoka guidelines for the management of IPMN of the pancreas. Pancreatology 2017;17:738-753.

3. Vege SS, Ziring B, Jain R, Moayyedi P; Clinical Guidelines Committee; American Gastroenterology Association. American gastroenterological association institute guideline on the diagnosis and management of asymptomatic neoplastic pancreatic cysts. Gastroenterology 2015;148:819-822.

4. European Study Group on Cystic Tumours of the Pancreas. European evidence-based guidelines on pancreatic cystic neoplasms. Gut 2018;67:789-804.

5. Del Chiaro M, Verbeke C, Salvia R, et al. European experts consensus statement on cystic tumours of the pancreas. Dig Liver Dis 2013;45:703-711.

6. Kim TH, Woo YS, Chon HK, et al. Predictors of malignancy in "pure" branch-duct intraductal papillary mucinous neoplasm of the pancreas without enhancing mural nodules on CT imaging: a nationwide multicenter study. Gut Liver 2018;12:583-590.

7. Kim TH, Song TJ, Hwang JH, et al. Predictors of malignancy in pure branch duct type intraductal papillary mucinous neoplasm of the pancreas: a nationwide multicenter study. Pancreatology 2015;15:405-410

8. Kobayashi G, Fujita N, Maguchi H, et al. Natural history of branch duct intraductal papillary mucinous neoplasm with mural nodules: a Japan Pancreas Society multicenter study. Pancreas 2014;43: 532-538.

9. Ohno E, Itoh A, Kawashima H, et al. Malignant transformation of branch duct-type intraductal papillary mucinous neoplasms of the pancreas based on contrast-enhanced endoscopic ultrasonography morphological changes: focus on malignant transformation of intraductal papillary mucinous neoplasm itself. Pancreas 2012;41: 855-862.

10. Kurihara N, Kawamoto H, Kobayashi Y, et al. Vascular patterns in nodules of intraductal papillary mucinous neoplasms depicted under contrast-enhanced ultrasonography are helpful for evaluating malignant potential. Eur J Radiol 2012;81:66-70. 